\title{
Complex Coil Assisted Single Coil Embolization for Small Intracranial Aneurysm
}

\author{
Ming-Shiang Yang, MD', ${ }^{1,}$ Tzu-Hsien Yang, MD³, Chang-Hsien Ou, MD', Si-Wa Chan, MD, \\ Tai-I Chen, MD ${ }^{6}$, Chia-Jung Yang, MD', Chia-Ming Chiang, MD', Wen-Chien Huang, MD7,8
}

The purpose of the technical note is to introduce the complex coil assisted coil embolization method in the treatment of intracranial small aneurysm, in order to enhance the safety of the procedure. The first microcatheter was navigated into the aneurysm sac and the ultrasoft coil was used as the embolization coil. If the embolizations coil could not stay within the aneurysm sac smoothly, such as coil herniation into parent artery during the delivery process. The second microcatheter would be navigated to the aneurysm level in the parent artery. Another complex coil was delivered within the parent artery via the second microcatheter to provide the neck bridge effect in order to enhance the stability of embolization coil. Besides, the protection coil will not disturb the parent artery flow. While the embolization coil was put into the aneurysm sac smoothly under the help of complex protective coil, the protective coil was then withdrawn gently. We use the most magnified view, dual-plane approach simultaneously to observe the stability of embolization coil. The embolization coil would be detached without any evidence of coil motion or vibration. The new method could provide the physiological protective method, without leaving any protective device such as stent within the parent artery.

Key Words : Complex coil; Aneurysm, Ruptured/therapy; Embolization, Therapeutic/methods Intracranial aneurysm

\footnotetext{
'Department of Radiology, Taitung Mackay Memorial Hospital, Taitung, Taiwan

2Department of Radiology, China Medical University Hospital, Taichung, Taiwan

${ }^{3}$ Department of Radiology, Chia-Yi Christian Hospital, Chia-Yi, Taiwan

${ }^{4}$ Department of Radiology, E-DA hospital, Kaohsiung, Taiwan

${ }^{5}$ Department of Radiology, Veteran General Hospital-Taichung, Taichung, Taiwan

${ }^{6}$ Department of Radiology, Show-Tran general hospital, Changhua, Taiwan

${ }^{7}$ Institute of Traditional Medicine, School of Medicine, National Yang-Ming University, Taipei, Taiwan

${ }^{8}$ Department of Surgery, Taipei Mackay Memorial Hospital, Taipei, Taiwan

Received July 19, 2013; accepted after revision August 9, 2013.

Correspondence to: Ming-Shiang Yang, MD, Department of Radiology, Taitung Mackay Memorial Hospital, Taitung, Taiwan, 1, lane 303, Changsha Street, Taitung, Taiwan

Tel. 886-89-310150 ext 345 Fax. 886-89-346482 E-mail: onecowyang2@gmail.com

This is an Open Access article distributed under the terms of the Creative Commons Attribution Non-Commercial License (http://creativecommons.org/licenses/by-nc/3.0) which permits unrestricted non-commercial use, distribution, and reproduction in any medium, provided the original work is properly cited.
} 
Small intracranial aneurysms $(\leqq 3.0 \mathrm{~mm})$ were found to be 5 times as likely to be associated with procedure-related rupture as larger aneurysms [1]. Such conditions pose technical challenges and treatment dilemmas $[2,3]$. Single coil embolization technique was introduced by Yang et al. [4] in 2009, via the usage the slight undersized coil (equivalent to neck size or 0.5 $\mathrm{mm}$ smaller than the aneurysm diameter), in order to enhance the safety during the embolization of very small sized intracranial aneurysm. Theoretically, smaller aneurysms are associated with higher risk of aneurysm rupture because random or accidental displacement of endovascular devices even by a few millimeters, which would be trivial with a large aneurysm, may lead to catastrophic rupture in the more confined lumen of a small aneurysm [2].

Easy coil protrusion and herniation into the parent artery was frequently found during the embolization procedure. We describe the complex coil assisted single coil embolization technique, to enhance the safety of the embolization procedure, provide the physiological protective technique without the necessary to leave any stent device within the parent artery.

\section{TECHNICAL REPORT}

\section{Materials and Results}

From 2009 January, total five patients received the complex coil assisted single coil embolization technique. The clinical and basic date was summarized in Table 1 . The technical success rate is $100 \%$, and no evidence of procedure related complication could be defined in the post-procedure bedside visit and following images.

\section{Illustrative Case and Technical Note in Patient 2}

The 43 years old female suffered from explosive headache. CTA (computed tomography angiography) and DSA (digital subtraction angiography) revealed carotid cave aneurysm growth, left lesion $5.5 \mathrm{~mm}$ in depth and $2.5 \mathrm{~mm}$ in neck, right lesion is $2.5 \mathrm{~mm}$ in depth and $2.5 \mathrm{~mm}$ in neck (Fig. 1A). Left lesion was embolized under the standard aneurysm embolization technique with GDC (Guglielmi detachable coil) coils.

Right lesion was done under the single coil embolization technique initially, with the usage of 0.010 microcatheter and Transcend microwire (Target Therapeutics/Boston Scientific, Fremont, CA, USA), but failed to put the embolization coil, $2 \times 8 \mathrm{~cm}$ Ultrasoft coil (Target Therapeutics/Boston Scientific, Fremont, CA, USA), into the aneurysm sac successfully.

Then we use the complex coil assisted single coil embolization technique as the following steps.

Step 1: The first microcather was navigated to the aneurysm sac and initiate the coiling process, but easy coil herniation was noted. The second microcatheter, was advanced to right ICA at the aneurysm level (Fig. 1B).

Step 2: The Complex coil (MicroVention, Aliso Viejo, CA, USA), $5 \times 12 \mathrm{~cm}$, was delivered as the protective coil within the parent artery at the aneurysm level, to act the neck bridge effect (Fig. 1C).

Step 3: The embolization coil was put into the aneurysm sac successfully with the help of protective coil, act as the neck bridge (Fig. 1D).

Step 4: The protective coil was withdrawn gently and observes the stability of embolization coil (Fig. 1E).

Step 5: After the protective coil was withdrawn completely, we use the most magnified views, dualplane approach simultaneously to observe the coil stability. It would be detached without any evidence of coil motion or vibration. Post embolization DSA revealed complete obliteration of the aneurysm sac

Table 1. The Summary of Basic Data

\begin{tabular}{lccccc}
\hline Patient No. & Age/Sex & S/S & Size/Neck/Location & Embolization coil & Protection coil \\
\hline 1 & $53 / F$ & SAH & $2.0 \mathrm{~mm} / 2.0 \mathrm{~mm} /$ Supraclinoid ICA & $2 \mathrm{~mm} \times 4 \mathrm{~cm}$ Ultrasoft coil & $4 \mathrm{~mm} \times 10 \mathrm{~cm}$ Complex coil \\
2 & $42 / \mathrm{F}$ & Explosive headache & $2.5 \mathrm{~mm} / 2.5 \mathrm{~mm} /$ Carotid cave & $2 \mathrm{~mm} \times 8 \mathrm{~cm}$ Ultrasoft coil & $5 \mathrm{~mm} \times 12 \mathrm{~cm}$ complex coil \\
3 & $64 / \mathrm{F}$ & Cranial nerve palsy & $2.5 \mathrm{~mm} / 2.5 \mathrm{~mm} /$ PcomA & $2 \mathrm{~mm} \times 8 \mathrm{~cm}$ Ultrasoft coil & $5 \mathrm{~mm} \times 12 \mathrm{~cm} \mathrm{Complex} \mathrm{coil}$ \\
4 & $59 / F$ & SAH & $2.0 \mathrm{~mm} / 2.0 \mathrm{~mm} / \mathrm{ICA}$ & $2 \mathrm{~mm} \times 2 \mathrm{~cm}$ Hypersoft coil & $5 \mathrm{~mm} \times 12 \mathrm{~cm} \mathrm{Complex} \mathrm{coil}$ \\
5 & $59 / F$ & SAH & $2.0 \mathrm{~mm} \times 2.0 \mathrm{~mm} /$ AcomA & $2 \mathrm{~mm} \times 3 \mathrm{~cm}$ Hypersoft coil & $3 \mathrm{~mm} \times 7 \mathrm{~cm} \mathrm{Complex} \mathrm{coil}$ \\
\hline
\end{tabular}

Abbreviations: S/S, sign and symptom; F, female; SAH, Subarachnoid hemorrhage; ICA, internal carotid artery; PcomA, posterior communicating artery 


\section{Coil Assisted Embolization for Intracranial Aneurysm}

(Fig. 1F).

Post-procedure bedside visit revealed well condition, no evidence of procedure related complication.

\section{DISCUSSION}

The complication rates in the treatment of very small ruptured intracranial aneurysms $(\leqq 3.0 \mathrm{~mm})$ is higher in previous published data [1-3]. In Nguyen TN et al series [1], procedure related rupture rate is $11.7 \%$ in very small aneurysms and $2.3 \%$ in larger ones. So how to increase and enhance the safety during the embolization procedure for small intracranial aneurysm is of great concern for interventional neuroradiologist. Current methods included the balloon or stent assisted method etc. The balloon assisted embolization technique originally described by Moret et al. [5] has been used in the endovascular treatment of wide neck aneurysms or in aneurysms with an unfavorable neckto-fundus ratio in which a standard coil placement technique may be unsuccessful. Some well known potential complication with balloon assisted
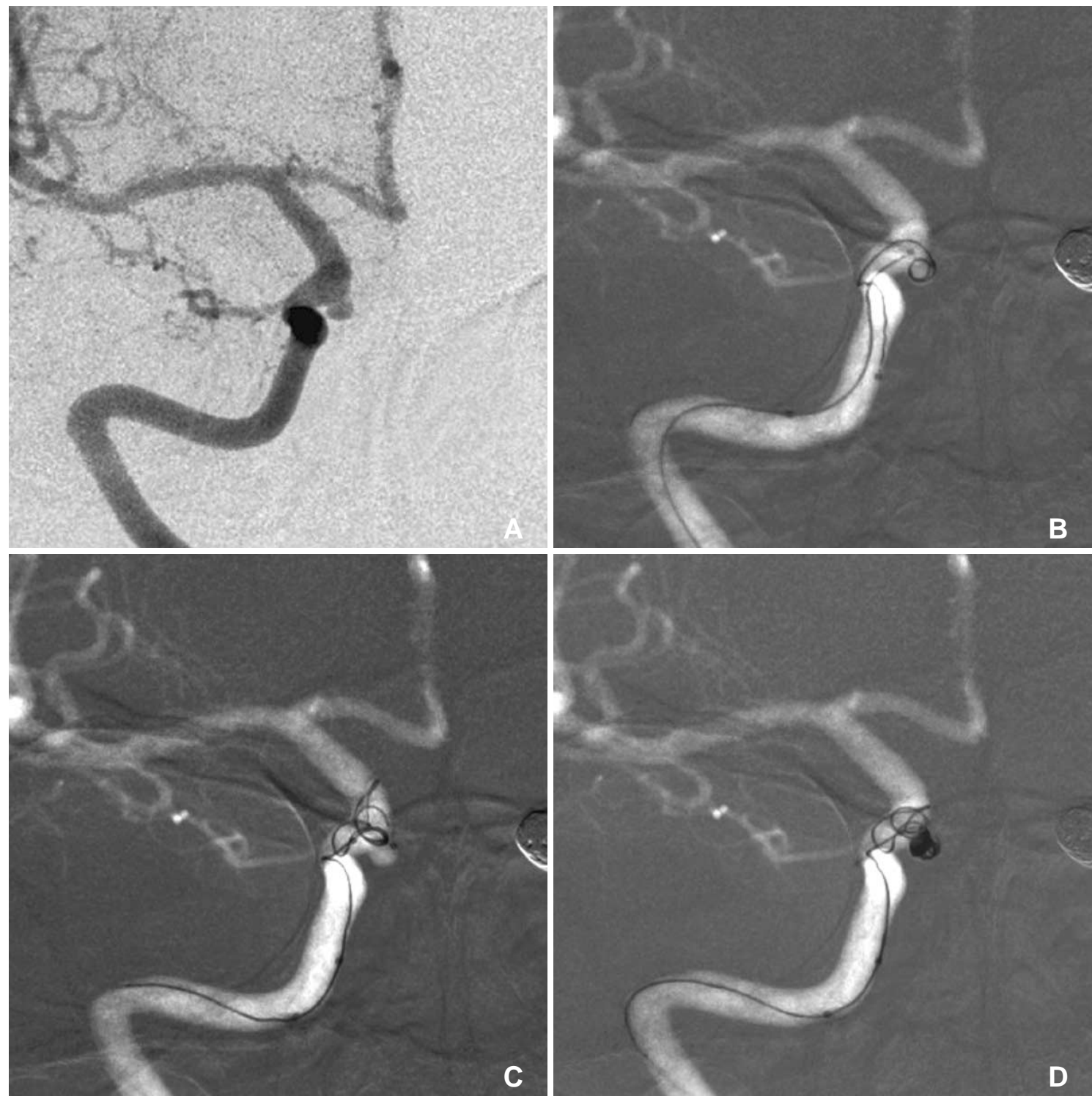

Fig. 1. A. the 43 years old female suffered from explosive headache. Digital subtraction angiography of right carotid artery revealed carotid cave aneurysm.

B. The first microcatheter was navigated to the aneurysm sac and initiates the coiling process, but easy coil herniation was noted. The second microcatheter was advanced to right ICA at the aneurysm level.

C. The Complex coil (MicroVention, Aliso Viejo, CA, USA), $5 \mathrm{~mm} \times 12 \mathrm{~cm}$, was delivered as the protective coil within the parent artery at the aneurysm level, to act the neck bridge effect.

D. The embolization coil was put into the aneurysm sac successfully with the help of protective coil, act as the neck bridge. 

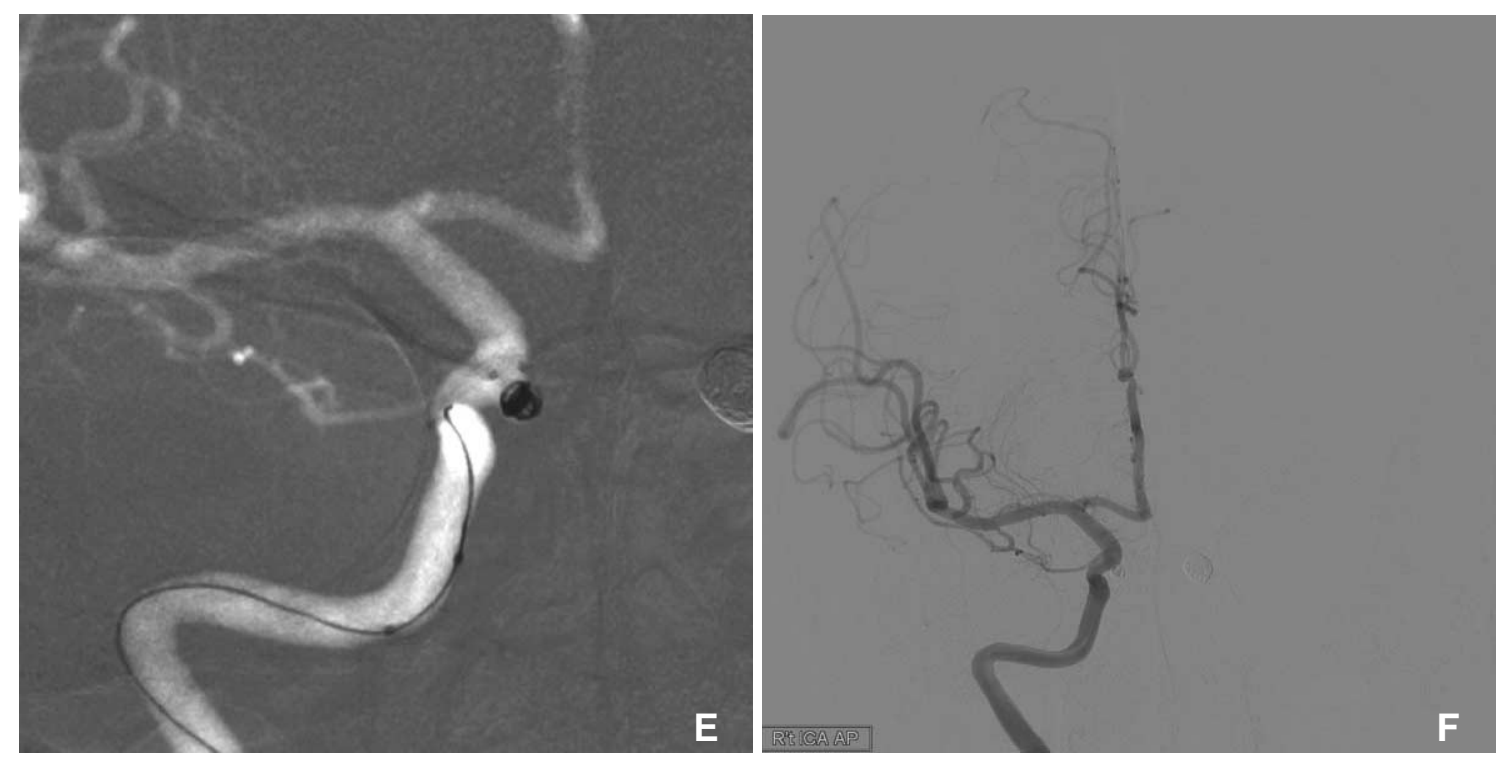

Fig. 1. E. The protective coil was withdrawn gently and observes the stability of embolization coil.

F. Post embolization DSA revealed complete obliteration of the aneurysm sac.

techniques, such as thromboembolic events, vasospasm, perforator occlusion, dissection, and ruptures of the parent artery or aneurismal sac, has been described in the literature [6]. Another drawback of the technique is the parent artery flow could be impaired during the balloon inflation period. Soeda et al. [7] investigated thromboembolic events associated with coil embolization with diffusion weighted images. They reported that embolic complications were more common in procedures utilizing balloon-assisted techniques than in procedures using conventional GDC techniques ( $73 \%$ vs. 50\%). Another widely used technique is stent assisted method. It could provide the neck bridge effect; enhance the coil stability during the embolization procedure. But one drawback of such technique is the necessity to use antiplatelet medication and leave the stent device within the parent artery.

The new complex coil assisted single coil embolization technique could enhance the safety during the treatment of small intracranial aneurysm. The complex protective coil could provide the neck bridge effect and enhance the embolization coil stability. Such method could also maintain the physiological phenomenon during the procedure, not disturb the parent artery flow. The complex protective coil could be withdrawn smoothly after complete delivery of the embolization coil. It would not disturb the coil stability, not leave any protective device within the parent artery. The preliminary result of the new technique is encouraging. The technical success rate is $100 \%$, no patient suffered from procedure related complication, no occurrence of thromboembolism.

The single coil embolization could provide the protective effect although the relative low packing density. In Sorteberg et al. studies [8], a marked reduction in intra-aneurysm flow after placement with GDCs even without the presence of thrombosis. They had hydrodynamic effect. The hydrodynamic effect could reduce stress on the aneurysm wall, immediately after the insertion of coil; provide the protective effect against bleeding [8].

In the conclusion of Goddard JK et al. study [3], small intracranial aneurysms treated with a single GDC placement demonstrated satisfactory stability despite having a low average packing density. In Gobin et al. study [9] revealed similar result-partial coiling of the aneurysm even in giant aneurysm could result in slower and incomplete filling and washout in the aneurysm.

For single coil embolization, the stability is of great concern. We always delay detach the embolization coil about 5-10 minutes, used the most magnificent view, dual-plane approach simultaneously, to detect potential coil motion or vibration. The coil stability of our preliminary result is well; no evidence of coil motion, vibration or migration could be detected.

In conclusion, the complex coil assisted single coil embolization could enhance the safety during the embolization procedure of very small intracranial aneurysm. Delay detached method was also recommended, in order to make sure the stability of embolization coil. The method could provide the physiological protective method, without leaving any 


\section{Coil Assisted Embolization for Intracranial Aneurysm}

protective device within the parent artery, not disturb the parent artery flow.

\section{References}

1. Nguyen TN, Raymond J, Guilbert F, Roy D, Bérubé MD, Mahmoud M, et al. Association of endovascular therapy of very small ruptured aneurysms with higher rates of procedure-related rupture. J Neurosurg 2008;108:1088-1092

2. Cloft HJ, Kallmes DF. Cerebral Aneurysm Performation Complicating Therapy with Guglielmi Detachable Coils: A MetaAnalysis. AJNR Am J Neuroradiol 2002;23:1706-1720

3. Goddard JK, Moran CJ, Cross III DT. Absent Relationship between the Coil-Embolization Ratio in Small Aneurysms Treated with a single Detachable Coil and Outcomes. AJNR Am J Neuroradiol 2005;26:1916-1920

4. Yang MS, Wong HF, Yang TH. Alternative option in the treatment of very small ruptured intracranial aneurysms. Surgical Neurology 2009;:S2:41-46

5. Moret J, Cognard C, Weill A. The "remodeling technique" in the treatment of wide neck intracranial aneurysms: Angiographic results and clinical follow-up in 56 cases. Intervent Neuroradiol 1997;3:21-35

6. Nelson PK, Levy DI. Balloon-assisted coil embolization of widenecked aneurysms of the internal carotid artery: medium-termangiographic and clinical follow-up in 22 patients. AJNR Am J Neuroradiol 2001;22:19-26

7. Soeda A, Sakai N, Sakai H, Iihara K, Yamada N, Imakita S, et al. Thromboembolic events associated with Guglielmidetachable coil embolization of asymptomatic cerebral aneurysms: evaluation of 66 consecutive cases with use of diffusion-weighted MR imaging. AJNR Am J Neuroradiol 2003;24:127-132

8. Sorteberg A, Sorteberg W, Aagaard Beverly DL. Hemodynamic versus Hydrodynamic Effects of Guglielmi Detachable Coils on Intra-Aneurysmal Pressure and Flow at Varying Pulse Rate and Systemic Pressure. AJNR Am J Neuroradiol 2004;25:1049-1057

9. Gobin YP, Counord JL, Flaud P. In vitro study of haemodynamics in a giant saccular aneurysm model: influence of flow dynamics in the parent vessel and effects of coil embolisation. Neuroradiology 1994;36:530-536 